\title{
Prognosis Score System to Predict Survival for COVID-19 Cases: a Korean Nationwide Cohort Study
}

Sung-Yeon Cho ${ }^{1,2^{*}}$, MD, PhD; Sung-Soo Park ${ }^{1,3^{*}}, \mathrm{MD}$, PhD; Min-Kyu Song ${ }^{4,5}$, MD; Young Yi Bae ${ }^{1}$, RN; Dong-Gun Lee $^{1,2^{*}}, \mathrm{MD}, \mathrm{PhD}$; Dong-Wook Kim ${ }^{1,3^{*}}, \mathrm{MD}, \mathrm{PhD}$

${ }^{1}$ Catholic Hematology Hospital, College of Medicine, The Catholic University of Korea, Seoul, Republic of Korea

${ }^{2}$ Division of Infectious Diseases, Department of Internal Medicine, College of Medicine, The Catholic University of Korea, Seoul, Republic of Korea

${ }^{3}$ Division of Hematology, Department of Internal Medicine, College of Medicine, The Catholic University of Korea, Seoul, Republic of Korea

${ }^{4}$ Data Research Institute, YMDtech Inc, Seoul, Republic of Korea

${ }^{5}$ St. Mary's Gong-Gam Mental Health Clinic, Siheung-si, Gyeonggi-do, Republic of Korea

*these authors contributed equally

Corresponding Author:

Dong-Gun Lee, MD, PhD

Catholic Hematology Hospital, College of Medicine

The Catholic University of Korea

Seoul St. Mary's Hospital

222 Banpo-daero, Seocho-Gu

Seoul, 06591

Republic of Korea

Phone: 82222586003

Email:symonlee@catholic.ac.kr

\section{Abstract}

Background: As the COVID-19 pandemic continues, an initial risk-adapted allocation is crucial for managing medical resources and providing intensive care.

Objective: In this study, we aimed to identify factors that predict the overall survival rate for COVID-19 cases and develop a COVID-19 prognosis score (COPS) system based on these factors. In addition, disease severity and the length of hospital stay for patients with COVID-19 were analyzed.

Methods: We retrospectively analyzed a nationwide cohort of laboratory-confirmed COVID-19 cases between January and April 2020 in Korea. The cohort was split randomly into a development cohort and a validation cohort with a 2:1 ratio. In the development cohort $(\mathrm{n}=3729)$, we tried to identify factors associated with overall survival and develop a scoring system to predict the overall survival rate by using parameters identified by the Cox proportional hazard regression model with bootstrapping methods. In the validation cohort $(\mathrm{n}=1865)$, we evaluated the prediction accuracy using the area under the receiver operating characteristic curve. The score of each variable in the COPS system was rounded off following the log-scaled conversion of the adjusted hazard ratio.

Results: Among the 5594 patients included in this analysis, 234 (4.2\%) died after receiving a COVID-19 diagnosis. In the development cohort, six parameters were significantly related to poor overall survival: older age, dementia, chronic renal failure, dyspnea, mental disturbance, and absolute lymphocyte count $<1000 / \mathrm{mm}^{3}$. The following risk groups were formed: low-risk (score $0-2$ ), intermediate-risk (score 3), high-risk (score 4), and very high-risk (score 5-7) groups. The COPS system yielded an area under the curve value of 0.918 for predicting the 14-day survival rate and 0.896 for predicting the 28 -day survival rate in the validation cohort. Using the COPS system, 28-day survival rates were discriminatively estimated at $99.8 \%, 95.4 \%, 82.3 \%$, and $55.1 \%$ in the low-risk, intermediate-risk, high-risk, and very high-risk groups, respectively, of the total cohort $(P<.001)$. The length of hospital stay and disease severity were directly associated with overall survival $(P<.001)$, and the hospital stay duration was significantly longer among survivors (mean 26.1, SD 10.7 days) than among nonsurvivors (mean 15.6, SD 13.3 days).

Conclusions: The newly developed predictive COPS system may assist in making risk-adapted decisions for the allocation of medical resources, including intensive care, during the COVID-19 pandemic. 


\section{KEYWORDS}

COVID-19; length of stay; mortality; prognosis; triage; digital health; prediction; cohort; risk; allocation; disease management; intensive care; decision making

\section{Introduction}

Since the outbreak of unexplained pneumonia in Wuhan, China, in December 2019, which was subsequently identified as COVID-19 caused by the newly discovered pathogen SARS-CoV-2, the COVID-19 pandemic remains active in over 180 countries [1,2]. Globally, as of November 6, 2020, $1,231,017$ of a total of $48,534,508$ patients with COVID-19 have died, representing an overall infection fatality rate of $2.54 \%$ $[3,4]$. The clinical spectrum of COVID-19 includes asymptomatic or presymptomatic, upper and lower respiratory tract infections and acute respiratory distress syndrome [5-8]. Although the majority of viral infections are self-limiting, COVID-19 cases that are severe (ie, dyspnea, hypoxia, or $>50 \%$ of lung involvement observed on imaging within 24-48 h) or critical (ie, respiratory failure, shock, or multiple organ failure) are of global concern and require medical resources for intensive care. The proportion of severe or critical COVID-19 cases and the corresponding case fatality rates vary by region and country, ranging from $10 \%-30 \%$ [8-10] to $2 \%-10 \%$, respectively $[3,4]$.

Most severe or critical cases occur in older patients or those with underlying comorbidities such as cancer, chronic obstructive pulmonary diseases, heart failure, or diabetes [11-13]. The clinical course of patients with COVID-19 also depends on multiple factors, including the immune status of the host, viral load of SARS-CoV-2, genetic diversity of the virus, and underlying diseases. However, details of viral factors and the host immune status (eg, cytokines released) are difficult to analyze in a real-world setting. Therefore, prognosis prediction systems should comprise basic factors such as initial symptoms at diagnosis, vital signs, hemogram parameters, and major underlying comorbidities.

As the global pandemic continues, the ability to detect, in a timely manner, patients with COVID-19 who are at a high risk of death and provide them with intensive care is important. Accordingly, the assessment of disease severity or mortality probability can be used to establish a sustainable strategy. Therefore, in this study, we aimed to develop an easy and simple scoring system that can predict COVID-19 mortality according to the patient's initial presentation and several major underlying comorbidities. Additionally, we investigated the association between the length of hospital stay and disease severity and survival status of patients with COVID-19.

\section{Methods}

\section{Study Design and Data Source}

This was a nationwide, retrospective cohort study on COVID-19 cases in Korea. For the purpose of this study, COVID-19 cases were defined based on laboratory confirmation of infection and positive results of SARS-CoV-2 reverse transcription polymerase chain reaction assays performed using the testing kits approved by the Korea Ministry of Food and Drug Safety, irrespective of the patient's clinical signs and symptoms [14,15]. Clinical data of patients in this cohort were managed by the Korea Disease Control and Prevention Agency (KDCA) and disclosed to the researchers after application and consent for research purposes in July 2020. The clinical and epidemiological information thus obtained included data of 5628 COVID-19 cases collected between January and April 2020. KDCA is an organization that aims to protect the Korean population from diseases, including emerging infectious diseases such as COVID-19, through national surveillance, health care research, and promotion of policies regarding disease prevention management. Patient data collected for this study included demographic and epidemiological characteristics, hemogram parameters at admission, maximal severity, and clinical outcome obtained from designated hospitals. Patients in the final cohort were randomly allocated to two subcohorts by using a random number generator: two-thirds into the "development cohort" and the remaining one-third into the "validation cohort." The predictive score was developed based on the development cohort, whereas the power of prediction was explored in the validation cohort.

This study was approved by the institutional review board of Seoul St. Mary's Hospital, Seoul, Korea (KC20ZADI0654). Individual patient consent was waived because the data retrieved were anonymous and publicly available.

\section{COVID-19 Management Setting}

In Korea, all suspected or confirmed cases of COVID-19 must be reported to the KDCA, as COVID-19 is regarded as a notifiable infectious disease. As a result, all patients with laboratory-confirmed COVID-19 were admitted to designated hospitals or residential treatment centers for isolation, monitoring of symptoms, and treatment. Clinical severity was classified into the following 8 levels according to patient performance, oxygen requirement, and organ failure [16]: (1) no limit of activity, (2) limited activity without oxygen supplementation, (3) requirement of oxygen supply with nasal cannula, (4) requirement of oxygen supply with facial mask, (5) requirement of noninvasive ventilation, (6) requirement of invasive ventilation, (7) requirement of extracorporeal membrane oxygenation (ECMO) for multiple organ failure, or (8) death. Death outcomes were evaluated regardless of maximum severity. Patients that required oxygen supply with invasive ventilation or ECMO were considered as invasive intensive care cases.

\section{Statistical Analysis}

Normally distributed numerical variables are presented as mean and SD values. Categorical variables are shown as absolute numbers and their proportions (n, \%). The hospital stay durations between two independent groups were compared using Student $t$ test. Overall survival was defined as the time from COVID-19 diagnosis to death due to any cause or up to the date of the last follow-up. Death events were censored at the time of hospital discharge for patients who were discharged. Overall survival 
rates at 14 days and 28 days were calculated using the Kaplan-Meier method and compared using the Log-rank test.

Within the development cohort, all risk factors with a $P$ value $<.05$ in the univariable analysis were entered into the multivariable model to identify factors associated with overall survival. Multivariable analysis was performed using the Cox proportional hazard regression model. We identified potential variables for the final prediction model based on 2000 bootstrap sampled datasets. When a parameter occurred in $60 \%$ or more of the bootstrap models, it was evaluated in the final multiple logistics regression model. We then computed the hazard ratios, 95\% CIs, and $P$ values for all metrics of the bootstrapped datasets in the final regression model. The final parameters used in the scoring system were defined by a $P$ value $<.05$ in the final regression model. To confirm the risk score for each significant parameter, we adjusted the hazard ratio values to a $\log _{\mathrm{e}}$ scale, followed by the conversion of the respective $\log _{\mathrm{e}}$ scale to a rounded integer point. In the validation cohort, the area under the receiver operating characteristic (AUROC) curve was measured to evaluate the prediction accuracy of the survival rates after 14 and 28 days. An AUROC value above 0.8 was considered reliable. Among the developed risk groups, we compared the length of hospital stay using one-way analysis of variance. For all statistical analyses, we used R statistical software (ver. 3.6.1, R Foundation for Statistical Computing). Statistical significance was set at $P<.05$.

\section{Results}

\section{Patient Characteristics}

In total, 5628 confirmed COVID-19 cases were reported between January and April 2020. Cases with a postmortem diagnosis $(n=7)$ or lack of clinical course data after diagnosis $(n=27)$ were excluded from the analysis. As shown in Figure 1, a total of 5594 patients with COVID-19 were included in this cohort. Overall, 41.2\% (2307/5594) of the patients were male, and 52.2\% (2919/5594) were aged 50 years or older. Baseline demographics are summarized in Table 1. In the total cohort, the most frequent age distribution was 50-59 years (1140/5594, $20.4 \%$ ), followed by $20-29$ years $(1110 / 5594,19.8 \%)$ and 60-69 years $(905 / 5594,16.2 \%)$. The most frequently reported symptoms included sputum $(1610 / 5594,28.8 \%)$, fever (1300/5594, 23.2\%), and dyspnea $(662 / 5594,11.8 \%)$. Common underlying comorbidities reported were hypertension $(1196 / 5594,21.4 \%)$ and diabetes $(686 / 5594,12.3 \%)$. Moreover, $4 \%(224 / 5594)$ of the patients had dementia and $3.2 \%$ (179/5594) of them had cardiac diseases. Distribution of these variables between the development $(n=3729)$ and validation $(\mathrm{n}=1865)$ cohorts is presented in Table 1.

Figure 1. Flow diagram of the nationwide cohort of patients with COVID-19.

\section{Patient data collected between January 2020 and April $2020(n=5628)$}

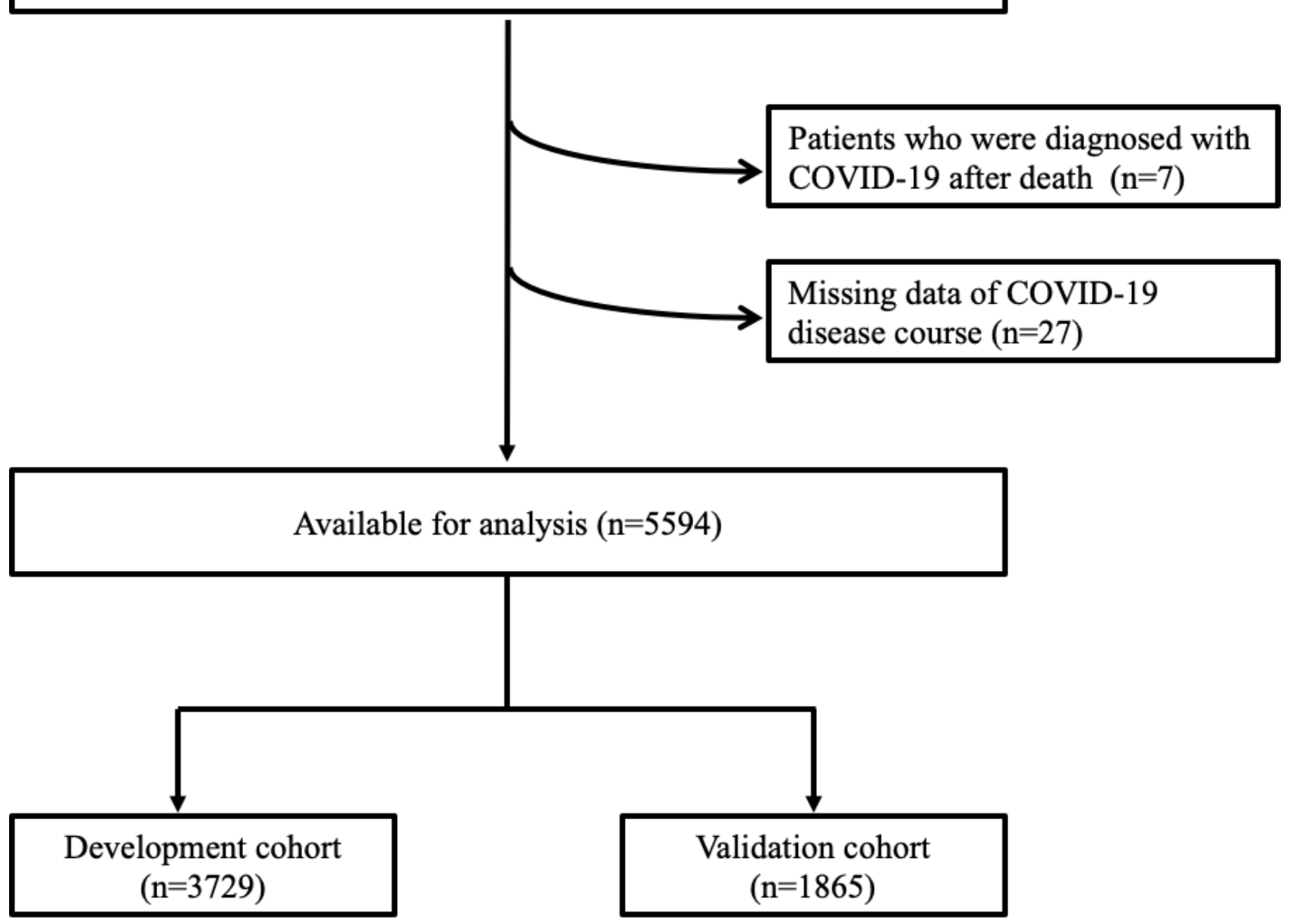


Table 1. Demographics of the study cohorts. All values presented in the table represent data collected at the time of COVID-19 diagnosis.

\begin{tabular}{|c|c|c|c|}
\hline \multirow[t]{2}{*}{ Variable } & \multirow[t]{2}{*}{ Total cohort $(\mathrm{N}=5594), \mathrm{n}(\%)$} & \multicolumn{2}{|l|}{ Subcohorts } \\
\hline & & $\begin{array}{l}\text { Development cohort } \\
(\mathrm{n}=3729), \mathrm{n}(\%)\end{array}$ & $\begin{array}{l}\text { Validation cohort } \\
(\mathrm{n}=1865), \mathrm{n}(\%)\end{array}$ \\
\hline \multicolumn{4}{|l|}{ Demographics, n (\%) } \\
\hline \multicolumn{4}{|l|}{ Age (years) } \\
\hline $0-9$ & $66(1.2)$ & $46(1.2)$ & $20(1.1)$ \\
\hline $10-19$ & $205(3.7)$ & $146(3.9)$ & $59(3.2)$ \\
\hline $20-29$ & $1,110(19.8)$ & $725(19.4)$ & $385(20.6)$ \\
\hline $30-39$ & $564(10.1)$ & $378(10.1)$ & $186(10)$ \\
\hline $40-49$ & $739(13.2)$ & $487(13.1)$ & $252(13.5)$ \\
\hline $50-59$ & $1,140(20.4)$ & $768(20.6)$ & $372(19.9)$ \\
\hline $60-69$ & $905(16.2)$ & $605(16.2)$ & $300(16.1)$ \\
\hline $70-79$ & $542(9.7)$ & $364(9.8)$ & $178(9.5)$ \\
\hline$\geq 80$ & $323(5.8)$ & $210(5.6)$ & $113(6.1)$ \\
\hline Gender (male) & $2,307(41.2)$ & $1536(41.2)$ & $1094(58.7)$ \\
\hline \multicolumn{4}{|l|}{ Comorbidity, n (\%) } \\
\hline Hypertension (missing $\mathrm{n}=3$ ) & $1196(21.4)$ & $795(21.3)$ & $401(21.5)$ \\
\hline Diabetes (missing $\mathrm{n}=3$ ) & $686(12.3)$ & $452(12.1)$ & $234(12.5)$ \\
\hline Dementia (missing $\mathrm{n}=329$ ) & $224(4)$ & $150(4)$ & $74(4)$ \\
\hline Cardiac disease (missing $n=19$ ) & $179(3.2)$ & $122(3.3)$ & $57(3.1)$ \\
\hline Cancer in active treatment ${ }^{\mathrm{a}}$ (missing $\mathrm{n}=4$ ) & $143(2.6)$ & $90(2.4)$ & $53(2.8)$ \\
\hline Asthma (missing $n=3$ ) & $128(2.3)$ & $82(2.2)$ & $46(2.5)$ \\
\hline Chronic hepatic disease $^{\mathrm{b}}$ (missing $\mathrm{n}=326$ ) & $82(1.5)$ & $47(1.3)$ & $35(1.9)$ \\
\hline Heart failure (missing $n=3$ ) & $58(1)$ & $36(1)$ & $22(1.2)$ \\
\hline Chronic renal failure (missing $n=3$ ) & $55(1)$ & $36(1)$ & $19(1)$ \\
\hline Chronic obstructive lung disease (missing $n=3$ ) & $40(0.7)$ & $25(0.7)$ & $15(0.8)$ \\
\hline Autoimmune disease (missing $\mathrm{n}=332$ ) & $38(0.7)$ & $31(0.8)$ & $7(0.4)$ \\
\hline \multicolumn{4}{|l|}{ Symptoms (missing $n=4), n(\%)$} \\
\hline Sputum & $1610(28.8)$ & $1114(29.9)$ & $496(26.6)$ \\
\hline Fever & $1300(23.2)$ & $852(22.8)$ & $448(24)$ \\
\hline Dyspnea & $662(11.8)$ & $454(12.2)$ & $208(11.2)$ \\
\hline Diarrhea & $516(9.2)$ & $345(9.3)$ & $171(9.2)$ \\
\hline Nausea or vomiting & $244(4.4)$ & $168(4.5)$ & $76(4.1)$ \\
\hline Fatigue & $233(4.2)$ & $164(4.4)$ & $69(3.7)$ \\
\hline Mental disturbance, $\mathrm{n}(\%)$ & $32(0.6)$ & $22(0.6)$ & $10(0.5)$ \\
\hline \multicolumn{4}{|c|}{ Systolic blood pressure (mmHg) (missing $n=135), n(\%)$} \\
\hline$<120$ & $1306(23.3)$ & $907(24.3)$ & $399(21.4)$ \\
\hline $120-129$ & $1138(20.3)$ & $733(19.7)$ & $405(21.7)$ \\
\hline $130-139$ & $1084(19.4)$ & $705(18.9)$ & $379(20.3)$ \\
\hline $140-159$ & $1418(25.3)$ & $960(25.7)$ & $458(24.6)$ \\
\hline$\geq 160$ & $513(9.2)$ & $330(8.8)$ & $183(9.8)$ \\
\hline \multicolumn{4}{|c|}{ Diastolic blood pressure (mmHg) (missing $n=135), \mathbf{n}(\%)$} \\
\hline$<80$ & $2102(37.6)$ & $1401(37.6)$ & $701(37.6)$ \\
\hline
\end{tabular}




\begin{tabular}{|c|c|c|c|}
\hline \multirow[t]{2}{*}{ Variable } & \multirow[t]{2}{*}{ Total cohort $(\mathrm{N}=5594), \mathrm{n}(\%)$} & \multicolumn{2}{|l|}{ Subcohorts } \\
\hline & & $\begin{array}{l}\text { Development cohort } \\
(\mathrm{n}=3729), \mathrm{n}(\%)\end{array}$ & $\begin{array}{l}\text { Validation cohort } \\
(\mathrm{n}=1865), \mathrm{n}(\%)\end{array}$ \\
\hline $80-89$ & $1797(32.1)$ & $1201(32.2)$ & $596(32)$ \\
\hline $90-99$ & $1056(18.9)$ & $686(18.4)$ & $370(19.8)$ \\
\hline$\geq 100$ & $504(9)$ & $347(9.3)$ & $157(8.4)$ \\
\hline Heart rate (per min) (missing $n=122$ ), mean (SD) & $85.8(15.1)$ & $85.8(15.1)$ & $85.8(15.1)$ \\
\hline$<110, \mathrm{n}(\%)$ & $5136(91.8)$ & $3,374(90.5)$ & 1709 (91.6) \\
\hline$\geq 110, \mathrm{n}(\%)$ & $336(6)$ & $272(7.3)$ & $117(6.3)$ \\
\hline Body temperature $\left({ }^{\circ} \mathrm{C}\right)($ missing $n=37)$, mean $(\mathrm{SD})$ & $36.9(0.6)$ & $36.9(0.6)$ & $36.9(0.6)$ \\
\hline$<38^{\circ} \mathrm{C}, \mathrm{n}(\%)$ & $5348(95.6)$ & $3523(94.5)$ & $1752(93.9)$ \\
\hline$\geq 38^{\circ} \mathrm{C}, \mathrm{n}(\%)$ & $209(3.7)$ & $179(4.8)$ & $103(5.5)$ \\
\hline \multicolumn{4}{|l|}{ Baseline hemogram } \\
\hline Hemoglobin (g/dL) (missing $n=1519)$, mean (SD) & $13.3(1.8)$ & $13.3(1.7)$ & $13.3(1.8)$ \\
\hline$\geq 12.5, \mathrm{n}(\%)$ & $2882(51.5)$ & $1923(51.6)$ & $959(51.4)$ \\
\hline$<12.5, \mathrm{n}(\%)$ & $1193(21.3)$ & $773(20.7)$ & $420(22.5)$ \\
\hline $\begin{array}{l}\text { Absolute lymphocyte count (per } \mathbf{m m}^{\mathbf{3}} \text { ) (missing } \\
\mathrm{n}=1542) \text {, mean (SD) }\end{array}$ & $1691(1,054)$ & $1697(955)$ & $1,681(1,225)$ \\
\hline$\geq 1000, \mathrm{n}(\%)$ & $3266(58.4)$ & $2161(58)$ & $1105(59.2)$ \\
\hline$<1000, \mathrm{n}(\%)$ & $786(14.1)$ & $518(13.9)$ & $268(14.4)$ \\
\hline $\begin{array}{l}\text { Platelet count (per } \mathbf{m m}^{3} \text { ) (missing } \mathbf{n}=1517 \text { ), mean } \\
\text { (SD) }\end{array}$ & $236,814(82,846)$ & $238,377(82,789)$ & $233,760(82,900)$ \\
\hline$\geq 100,000, \mathrm{n}(\%)$ & $3986(71.3)$ & 2634 (70.6) & $1352(72.5)$ \\
\hline$<100,000, \mathrm{n}(\%)$ & $91(1.6)$ & $62(1.7)$ & $29(1.6)$ \\
\hline Follow-up (days), median (95\% CI) & $25(24-25)$ & $25(24-25)$ & $25(24-25)$ \\
\hline
\end{tabular}

${ }^{\mathrm{a}}$ Cases that achieved complete cure of cancer were excluded.

${ }^{\mathrm{b}}$ Cases with chronic hepatitis were included in this category.

\section{Clinical Course, Outcome, and Length of Hospital Stay in the Total Cohort}

Among the 5594 patients included in the analysis, 234 (4.2\%) died after a COVID-19 diagnosis was made, resulting in a cohort case fatality rate of $4.2 \%$. Excluding death, the maximal clinical disease severity during hospitalization was as follows: (1) no limit of activity in $79.6 \%$ (4455/5594) of the patients, (2) limited activity without oxygen supplementation in 5.9\% (330/5594) of the patients, (3) requirement of oxygen supply with nasal cannula in $8.4 \%$ (469/5594) of the patients, (4) requirement of oxygen supply with facial mask or advanced device (such as noninvasive ventilation or high flow oxygen therapy) in $1.4 \%$ (76/5594) of the patients, and (5) requirement of invasive intensive care such as invasive ventilation for acute respiratory distress syndrome or ECMO for multiple organ failure in $0.5 \%$ (30/5594) of the patients (Figure 2).

Overall, the mean duration of hospital stay was 25.6 (SD 11.0) days. Hospital stay was significantly longer among survivors (mean 26.1, SD 10.7 days) than among nonsurvivors (mean 15.6, SD 13.3 days; $P<.001$ ). As shown in Figure 2, the higher the severity of clinical course among survivors, the longer was their hospital stay: no limit of activity, mean 25.4 (SD 10.2) days; limited activity without oxygen supplementation, mean 27.6 (SD 10.6) days; oxygen supply with nasal cannula, mean 29.8 (SD 12.0) days; oxygen supply with facial mask or advanced device, mean 32.5 (SD 14.0) days; and invasive intensive care groups, mean 41.0 (SD 15.4) days. 
Figure 2. Maximal COVID-19 disease severity and duration of hospital stay. Hospital stay duration differed significantly according to maximal disease severity; the mean length of hospital stay is indicated.

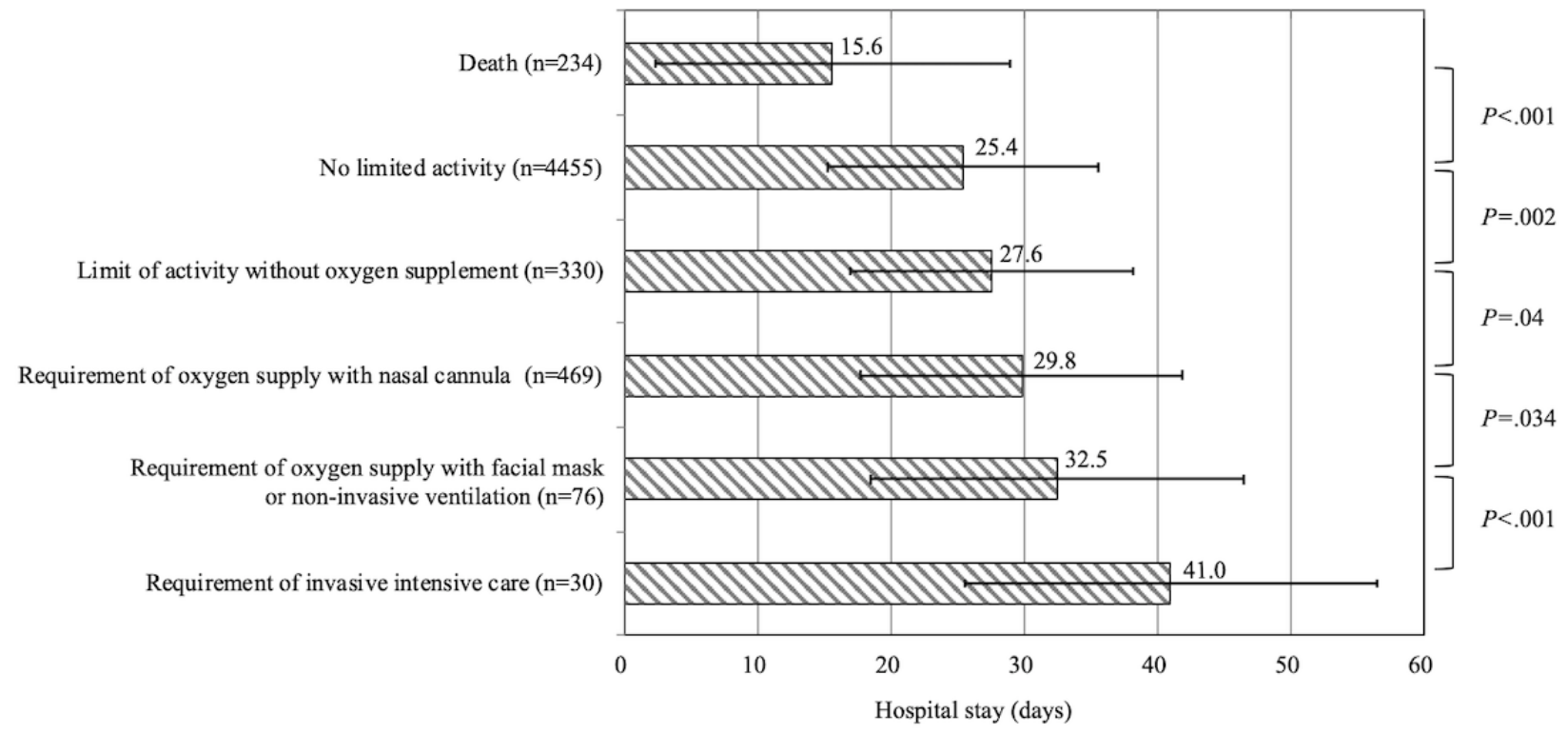

\section{Analysis of Factors Associated With Overall Survival in the Development Cohort}

The univariable analysis identified the following potential factors associated with poor overall survival: age ( $\geq 70$ or $50-69$ years vs <50 years); sex (male); comorbidities such as hypertension, diabetes, dementia, chronic cardiac disease, cancer in active treatment, chronic pulmonary disease, and chronic renal failure; dyspnea, fatigue, mental disturbance, high systolic blood pressure $(\geq 140 \mathrm{mmHg})$, low diastolic blood pressure $(<80$ $\mathrm{mmHg}$ ), tachycardia (heart rate $\geq 110 / \mathrm{min})$, and fever $\left(\geq 38^{\circ} \mathrm{C}\right)$ at the time of diagnosis; and cytopenia (hemoglobin level $<12.5$ $\mathrm{g} / \mathrm{dL}$, absolute lymphocyte count [ALC] $<1000 / \mathrm{mm}^{3}$, and platelet count $<100,000 / \mathrm{mm}^{3}$ ), as shown in Table 2 . 
Table 2. Univariable analysis for potential factors associated with overall survival in the development cohort. All values presented in the table represent data collected at the time of initial COVID-19 diagnosis.

\begin{tabular}{|c|c|c|c|c|}
\hline \multirow[t]{2}{*}{ Factor } & \multirow{2}{*}{$\begin{array}{l}\text { Number of patients } \\
(\mathrm{n}=3729), \mathrm{n}(\%)\end{array}$} & \multicolumn{2}{|c|}{ Overall survival rate, $\%(95 \% \mathrm{CI})$} & \multirow[t]{2}{*}{$P$ value } \\
\hline & & At 14 days & At 28 days & \\
\hline Age (years) & & & & $<.001$ \\
\hline$<50$ & $1782(47.8)$ & $99.9(99.8-100)$ & $99.8(99.5-100)$ & \\
\hline $50-69$ & $1373(36.8)$ & $98.6(98-99.2)$ & $98(97.2-98.8)$ & \\
\hline$\geq 70$ & $574(15.4)$ & $87(84.3-89.8)$ & $81.6(78.3-85)$ & \\
\hline Sex & & & & .01 \\
\hline Female & $2193(58.8)$ & $97.8(97.2-98.4)$ & $96.8(96-97.6)$ & \\
\hline Male & $1536(41.2)$ & $96.9(96-97.8)$ & $95.2(94.1-96.4)$ & \\
\hline \multicolumn{5}{|l|}{ Comorbidity } \\
\hline Hypertension & & & & $<.001$ \\
\hline No & $2932(78.6)$ & $98.7(98.3-99.1)$ & $98(97.4-98.6)$ & \\
\hline Yes & $795(21.3)$ & $92.7(90.9-94.6)$ & $89.7(87.4-91.9)$ & \\
\hline Diabetes & & & & $<.001$ \\
\hline No & $3275(87.8)$ & $98.3(97.8-98.7)$ & $97.3(96.7-98)$ & \\
\hline Yes & $452(12.1)$ & $91.5(88.9-94.1)$ & $88.1(85-91.3)$ & \\
\hline Dementia & & & & $<.001$ \\
\hline No & $3359(90.1)$ & $98.3(97.9-98.7)$ & $97.3(96.7-97.9)$ & \\
\hline Yes & $150(4)$ & $74.5(67.8-81.8)$ & $67.4(60.1-75.6)$ & \\
\hline Chronic cardiac disease $^{\text {a }}$ & & & & $<.001$ \\
\hline No & $3582(96.1)$ & $97.7(97.2-98.2)$ & $96.7(96-97.3)$ & \\
\hline Yes & $147(3.9)$ & $91.1(86.6-95.8)$ & $85.8(79.9-92)$ & \\
\hline Cancer in active treatment & & & & .03 \\
\hline No & $3636(97.5)$ & $97.5(97-98)$ & $96.3(95.7-97)$ & \\
\hline Yes & $90(2.4)$ & $95.5(91.2-99.9)$ & $89.8(82.5-97.7)$ & \\
\hline Chronic pulmonary disease ${ }^{b}$ & & & & $<.001$ \\
\hline No & $3628(97.3)$ & $97.6(97.1-98.1)$ & $96.5(95.9-97.2)$ & \\
\hline Yes & $101(2.7)$ & $92(86.8-97.5)$ & $85.3(77.9-93.3)$ & \\
\hline Chronic hepatic disease & & & & 6 \\
\hline No & $3463(92.9)$ & $97.3(96.7-97.8)$ & $96(95.2-96.7)$ & \\
\hline Yes & $47(1.3)$ & $97.9(93.8-100)$ & $95.3(89.1-100)$ & \\
\hline Chronic renal failure & & & & $<.001$ \\
\hline No & $3691(99)$ & $97.5(97-98)$ & $96.3(95.7-97)$ & \\
\hline Yes & $36(1)$ & $85.7(74.9-98.1)$ & $81.2(68.4-96.4)$ & \\
\hline Autoimmune disease & & & & .56 \\
\hline No & $3476(93.2)$ & $97.3(96.7-97.8)$ & $96(95.3-96.7)$ & \\
\hline Yes & $31(0.8)$ & $96.8(90.8-100)$ & $90.7(78.7-100)$ & \\
\hline \multicolumn{5}{|l|}{ Symptoms } \\
\hline Sputum & & & & .59 \\
\hline No & $2612(70)$ & $97.4(96.8-98.1)$ & $96(95.2-96.9)$ & \\
\hline Yes & $1114(29.9)$ & $97.4(96.4-98.3)$ & $96.5(95.4-97.7)$ & \\
\hline Dyspnea & & & & $<.001$ \\
\hline
\end{tabular}




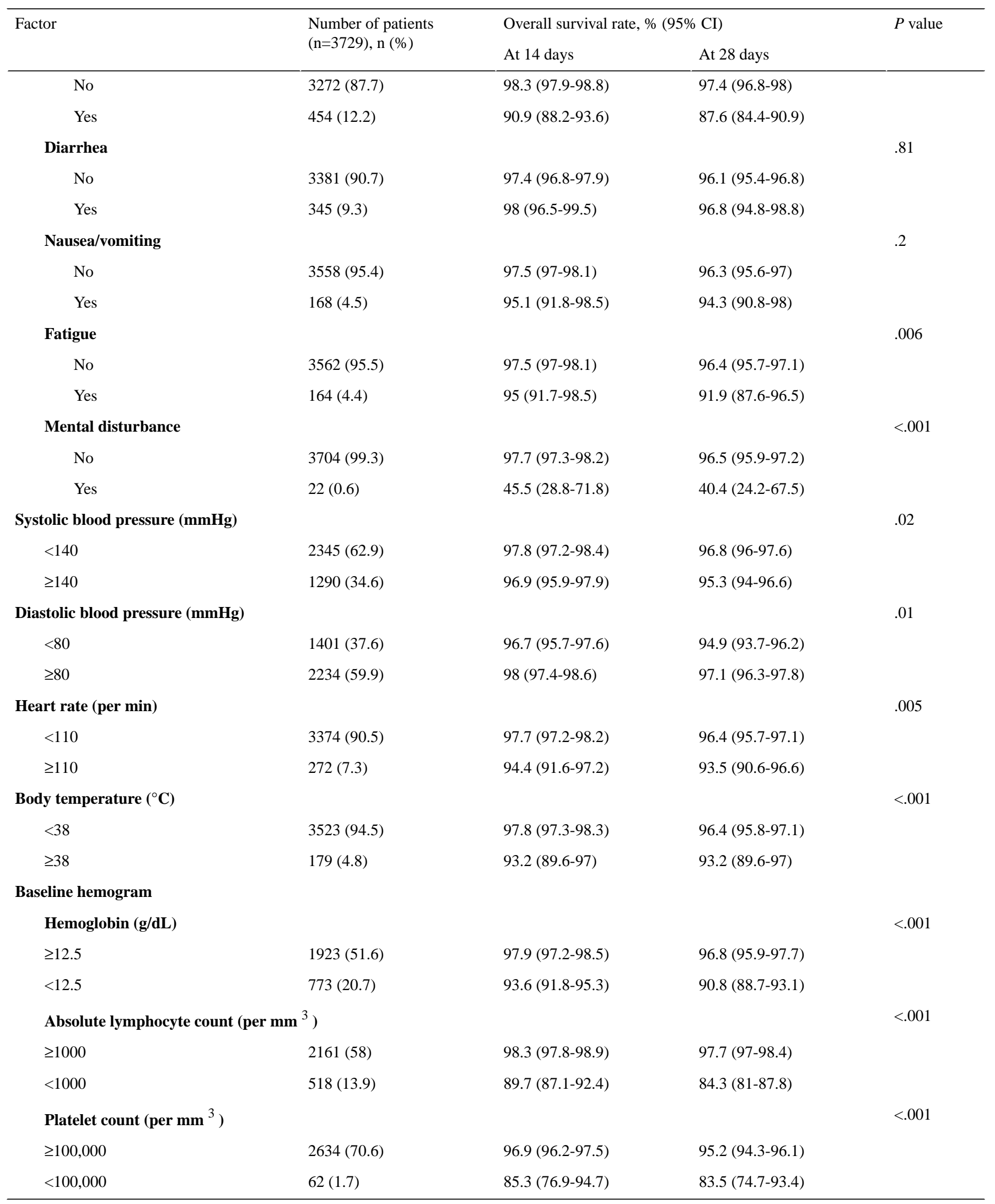

${ }^{\mathrm{a}}$ Chronic cardiac disease was a composite variable including heart failure and cardiac disease.

${ }^{\mathrm{b}}$ Chronic pulmonary disease was a composite variable including asthma and chronic obstructive lung disease. 


\section{COVID-19 Prognosis Score for Predicting Overall Survival}

In the bootstrap analysis, we identified that older age (50-69 or $\geq 70$ years) and comorbidities, including dementia, chronic renal failure, presentation of dyspnea, mental disturbance at diagnosis, and ALC $<1,000 / \mathrm{mm}^{3}$ were significantly associated with poor overall survival. Assigned risk scores obtained by rounding the $\log _{\mathrm{e}}$ scale of hazard ratio are shown in Table 3: age (50-69 years, 2 points; $\geq 70$ years, 3 points), underlying dementia (1 point), chronic renal failure (1 point), dyspnea (1 point), mental disturbance (1 point), ALC $<1000 / \mathrm{mm}^{3}$ (1 point). We determined the COVID-19 prognosis score (COPS) based on the risk scores obtained for each patient and summing the respective scores of the 6 parameters. The total COPS ranged between 0 and 8 .

We explored the clinical prediction score in the validation cohort using the AUROC curve analysis, which resulted in an AUROC value of 0.918 (95\% CI 0.91-0.927) for the 14-day overall survival rate and 0.896 (95\% CI 0.872-0.911) for the 28-day overall survival rate, indicating a reliable discrimination through the COPS system (Figure 3). Thereafter, we applied the scoring system to the total cohort, which resulted in a score range of 0-7 points (Figure 4A). This scoring system discriminately classified the patients into 8 groups. The 28 -day overall survival rates were predicted as $99.9 \%$ (95\% CI 99.7-100) in the 0-point group $(n=2348), 99.7 \%$ (95\% CI 99.1-100) in the 1-point group $(\mathrm{n}=317), 99.6 \%(95 \%$ CI $92.9-99.9)$ in the 2-point group $(\mathrm{n}=1511), 95.4 \%$ (95\% CI 93.9-97.1) in the 3-point group $(\mathrm{n}=815), 82.3 \%(95 \% \mathrm{CI} 78.5-86.4)$ in the 4-point group $(\mathrm{n}=395), 60 \%(95 \%$ CI 39.2-52.8) in the 5-point group $(\mathrm{n}=170)$, $32.9 \%$ (95\% CI 20.6-52.7) in the 6-point group $(\mathrm{n}=36)$, and $50 \%(95 \%$ CI 12.5-100) in the 7-point group $(n=2)(P<.001$; Figure 4A).

We then determined the risk groups based on the final COPS system; these included low-risk (0-2 points, $n=4167)$, intermediate-risk ( 3 points, $n=774$ ), high-risk ( 4 points, $n=321$ ), and very-high risk ( $\geq 5$ points, $n=98$ ) groups. The 28 -day overall survival rates for these groups were as follows: low-risk group, 99.8\% (95\% CI 99.6-99.9); intermediate-risk group, $95.4 \%$ (95\% CI 93.9-97.1); high-risk group, 82.3\% (95\% CI 78.5-86.4); and very-high risk group, $55.1 \%$ (95\% CI 48.5-62.5) $(P<.001$; Figure 4B). The developed COPS calculator is available online [17].

Furthermore, a significant increase in the length of hospital stay was observed as the risk group advanced: low-risk group, mean 25.4 (SD 10.4) days; intermediate-risk group, mean 27.2 (SD 10.9) days; and high-risk or very high-risk groups, mean 30.8 (SD 11.9) days $(P<.001)$. 
Table 3. The final scoring model in the development cohort. All values presented in the table represent data collected at the time of initial COVID-19 diagnosis.

\begin{tabular}{llll}
\hline Factor & Adjusted hazard ratio $(95 \% \mathrm{CI})^{\mathrm{a}}$ & $P$ value $^{\mathrm{a}}$ & $\log _{\mathrm{e}}$ value of hazard ratio \\
\hline $\begin{array}{l}\text { Age (years) } \\
<50\end{array}$ & 1 (reference) & $\mathrm{N} / \mathrm{A}^{\mathrm{b}}$ & 0 \\
$50-69$ & $6.7(1.09-43.93)$ & .047 & 1.831 \\
$\geq 70$ & $26.03(4.26-169.8)$ & $<.001$ & 3.186
\end{tabular}

Sex

$\begin{array}{ll}\text { Female } & 1 \text { (reference) } \\ \text { Male } & 1.35(0.85-2.15)\end{array}$

N/A N/A

N/A

Comorbidity

Hypertension

$\begin{array}{ll}\text { No } & 1 \text { (reference) } \\ \text { Yes } & 1.21(.75-1.94)\end{array}$

N/A

N/A

N/A

$1.21(.75-1.94)$

.48

N/A

N/A

Diabetes

$\begin{array}{ll}\text { No } & 1 \text { (reference) } \\ \text { Yes } & 1.71(1.08-2.7)\end{array}$

N/A

N/A

N/A

$.61 \quad$ N/A N/A

\section{Dementia}

No

Yes

1 (reference)

$3.92(2.33-6.61)$

N/A $\quad 0$

$<.001 \quad 1.35$

0

1

Chronic cardiac disease ${ }^{c}$

$\begin{array}{ll}\text { No } & 1 \text { (reference) } \\ \text { Yes } & 1.15(.61-2.15)\end{array}$

N/A

N/A

N/A

.57

N/A

N/A

Cancer in active treatment

1 (reference)

$1.83(.72-4.71)$

No

Yes

Chronic pulmonary disease ${ }^{\mathrm{d}}$

No

Yes

1 (reference)

$1.76(.857-3.625)$

Chronic renal failure

$\begin{array}{ll}\text { No } & 1 \text { (reference) } \\ \text { Yes } & 3.48(1.39-8.85)\end{array}$

Symptoms

Dyspnea
No

Yes

Fatigue

$\begin{array}{ll}\text { No } & 1 \text { (reference) } \\ \text { Yes } & .69(.3-1.57)\end{array}$

Mental disturbance

No

Yes

1 (reference)

$4.04(1.72-9.53)$

1 (reference)

$2.31(1.44-3.69)$

Systolic blood pressure (mmHg)

Final score

N/A

\section{N/A}

(n)




\begin{tabular}{|c|c|c|c|c|}
\hline Factor & Adjusted hazard ratio $(95 \% \mathrm{CI})^{\mathrm{a}}$ & $P$ value $^{\mathrm{a}}$ & $\log _{e}$ value of hazard ratio & Final score \\
\hline$<140$ & 1 (reference) & N/A & N/A & N/A \\
\hline$\geq 140$ & $.93(.57-1.54)$ & .59 & N/A & N/A \\
\hline
\end{tabular}

Diastolic blood pressure (mmHg)

\begin{tabular}{|c|c|c|c|}
\hline$<80$ & 1 (reference) & N/A & N/A \\
\hline$\geq 80$ & $1.03(.63-1.68)$ & .63 & N/A \\
\hline
\end{tabular}

Heart rate (per min)

$<110$

$$
\begin{aligned}
& 1 \text { (reference) } \\
& 1.88(.96-3.68)
\end{aligned}
$$$$
\geq 110
$$

N/A

Body temperature $\left({ }^{\circ} \mathrm{C}\right)$

$\begin{array}{ll}<38 & 1 \text { (reference) } \\ \geq 38 & .79(.39-1.63)\end{array}$

$.18 \quad \mathrm{~N} / \mathrm{A}$

N/A

aseline hemogram

Hemoglobin (g/dL)

$\begin{array}{ll}\geq 12.5 & 1 \text { (reference) } \\ <12.5 & 1.14(.71-1.83)\end{array}$

$\begin{array}{ll}\text { N/A } & \text { N/A } \\ .46 & \text { N/A }\end{array}$

N/A

N/A

bsolute lymphocyte count (per $\mathbf{m m}^{3}$ )

$\begin{array}{ll}\geq 1000 & 1 \text { (reference) } \\ <1000 & 2.71(1.66-4.43)\end{array}$

$2.71(1.66-4.43)$

N/A $\quad 0$

0

$<.001 \quad .982$

N/A

$.55 \quad$ N/A N/A

Platelet count (per $\mathbf{m m}^{3}$ )

\begin{tabular}{lllll}
$\geq 100,000$ & 1 (reference) & N/A & N/A & N/A \\
$<100,000$ & $1.68(.77-3.7)$ & .34 & N/A & N/A \\
\hline
\end{tabular}

${ }^{\text {a } B a s e d ~ o n ~} 2000$ bootstrap samples.

${ }^{\mathrm{b}}$ Not applicable.

${ }^{\mathrm{c}}$ Chronic cardiac disease was a composite variable including heart failure and cardiac disease.

${ }^{\mathrm{d}}$ Chronic pulmonary disease was a composite variable including asthma and chronic obstructive lung disease.

Figure 3. Receiver operating characteristics curve analysis of the newly developed COVID-19 prognosis score system in the validation cohort. A receiver operating characteristic curve analysis achieved an area under the curve value of (A) 0.918 (95\% CI, 0.91-0.927) for 14-day survival and (B) 0.896 (95\% CI 0.872-0.911) for 28-day survival.
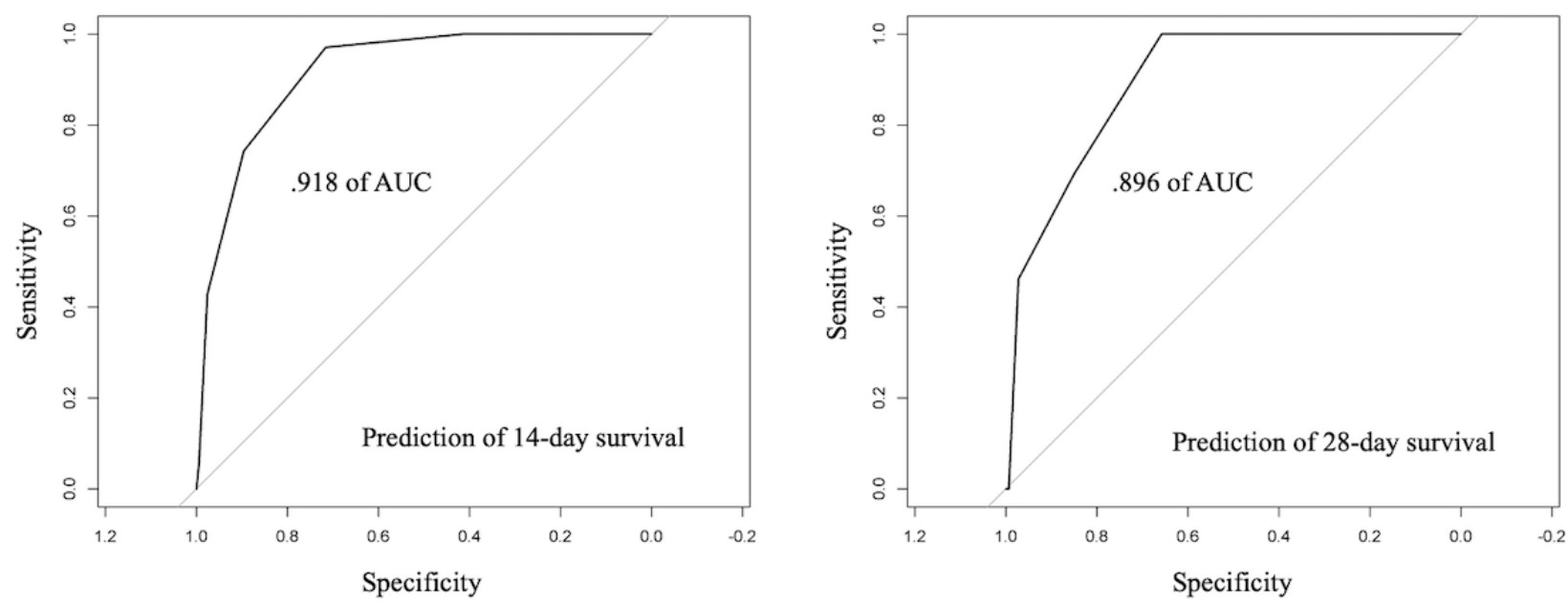
Figure 4. Probability of overall survival in patients with COVID-19 according to (A) each score and (B) the final COVID-19 prognosis score system.

(A)

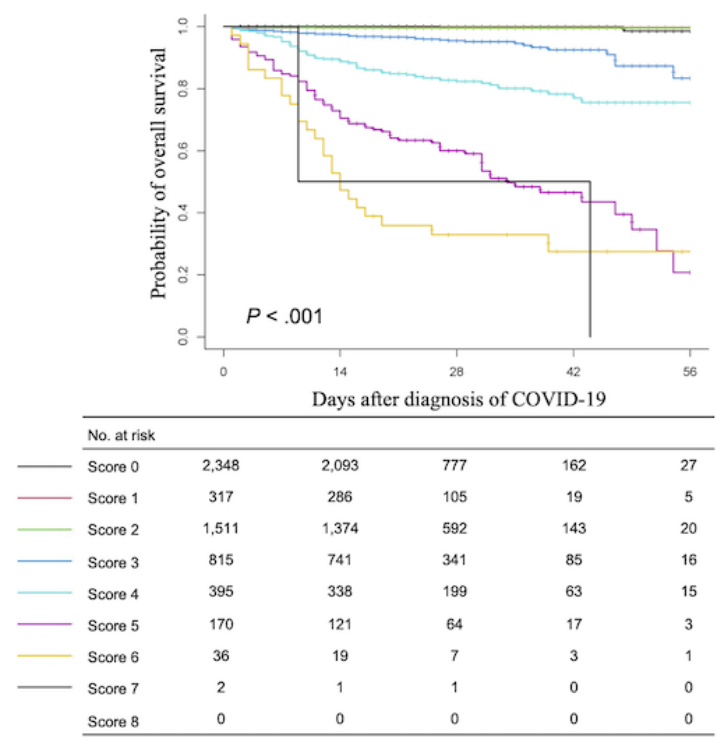

\section{Discussion}

In this study, we developed a new scoring system (COPS) to predict the mortality of patients with COVID-19 by using nationwide data of 5594 patients with COVID-19. The COPS system comprises basic demographics, initial symptoms, vital signs, and hemogram results at diagnosis. The risk score was stratified into four risk groups: low-risk, intermediate-risk, high-risk, and very high-risk groups associated with 28-day overall survival rate probabilities of $99.8 \%, 95.4 \%, 82.3 \%$, and $55.1 \%$, respectively. The AUROC curve analysis indicated that the prediction ability of the COPS system was excellent in the validation cohort.

Among the comorbidities identified in this study, dementia had the highest impact on mortality (adjusted hazard ratio 3.92) followed by chronic renal failure (adjusted hazard ratio 3.48). Moreover, the prognosis was poor when mental disturbance (adjusted hazard ratio 4.04) was noted at the time of diagnosis or when the patient had underlying dementia (adjusted hazard ratio 3.92). These two factors are related to the presence of SARS-CoV-2 infection clusters in nursing homes or long-term care facilities between February and April 2020 in Korea. Therefore, there is a need to establish screening and infection control strategies for long-term health care facilities [18-21].

In this study, an ALC of $<1000 / \mathrm{mm}^{3}$ was found to affect the survival rate of patients with COVID-19. ALC has been used as a prognostic factor for common respiratory viruses, including respiratory syncytial virus or other viral reactivation in immunocompromised hosts [22]. A recent meta-analysis reported that lymphopenia on admission was associated with poor outcomes in patients with COVID-19 [23]. Further immunological studies on patients with COVID-19 are needed to elucidate the mechanism of lymphopenia and $\mathrm{T}$ cell reactivation, as well as cytokines [24].

Regarding disease severity, approximately $87 \%$ of patients did not need oxygen supplementation. Approximately $10 \%$ of the
(B)

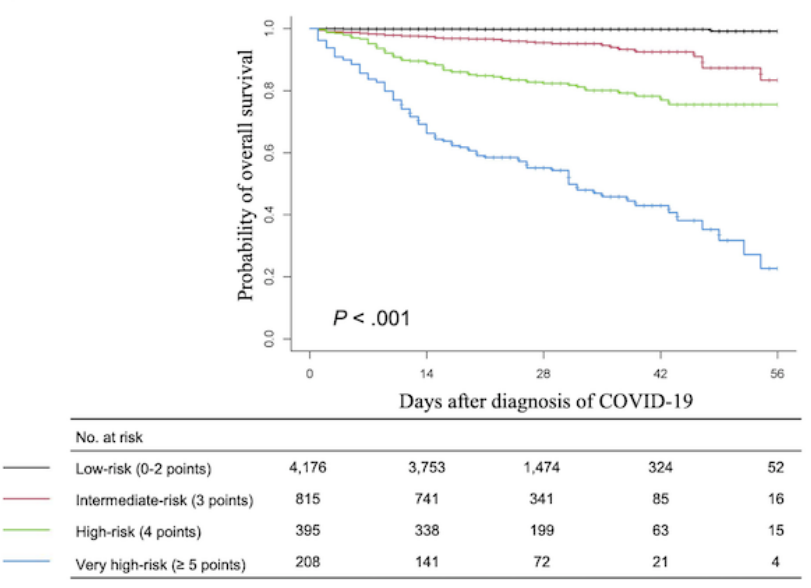

patients required oxygen supplementation; of these, 25\% received oxygen via a simple mask or mechanical ventilators. From the perspective of a national strategy for new infectious disease crisis, it is important to determine the proportion of critically ill patients and the length of hospital stay according to disease severity in order to prepare medical resources, such as critical care beds. This study found that the hospitalization period was significantly longer among survivors than among nonsurvivors. Moreover, among the survivors, the length of hospital stay was directly associated with disease severity.

In this cohort, the infection fatality rate after COVID-19 diagnosis was 4.18\% (234/5594). However, until April 30, 2020, the cumulative number of confirmed COVID-19 cases in Korea was 10,765 with 247 deaths, representing an actual mortality rate of $2.29 \%$ during the same period [25]. As of November 6, 2020, the total cumulative number of confirmed COVID-19 cases and deaths were 27,195 and 476, respectively, representing an infection fatality rate of $1.75 \%$ in Korea [25]. This disparity in mortality rates can be attributed to the fact that not all data were reported at the time this cohort was released. However, considering the large number of patients included in this cohort and collection of most deceased cases, the findings of this study are still meaningful and carry little statistical bias. In addition, remdesivir was not available in Korea during the study period, and infectious disease prevention and control measures were less established in the early phase of the COVID-19 pandemic. Thus, several mass infection episodes might have caused the relatively high mortality rate during the early phase of the pandemic in Korea.

Several studies have attempted to determine predictive factors for severe or fatal COVID-19 cases. A study on risk factors for fatal COVID-19 cases performed in China proposed a scoring system comprising age, procalcitonin, aspartate aminotransferase level, coronary heart disease, and cerebrovascular disease; this system was developed using data of 1590 inpatients with COVID-19 collected until January 2020. The nomogram showed discriminatory power with a $\mathrm{C}$-index of 0.91 to predict survival 
[13]. Another study analyzed the risk of intensive care unit admissions and deaths among 4997 individuals under investigation at an academic hospital in New York. The study used age, heart rate, procalcitonin, lactate dehydrogenase, heart failure, and chronic obstructive pulmonary disease to predict care in intensive care units and deaths, yielding an accuracy of 0.74 and 0.83 , respectively [26]. In the United Kingdom, a similar study was conducted on 17 million individuals included in the OpenSAFELY database, a near-real-time primary care patient record, which pseudonymously identified 10,926 COVID-19-related deaths. The study found male sex, older age and deprivation, diabetes, and severe asthma as significant risk factors for death [27]. However, the study did not analyze survival and death among patients with a confirmed COVID-19 diagnosis. In addition to studies attempting to predict death in patients with COVID-19, other studies have focused on severity index to predict severe or critical cases [10,28-31]. More recently, a machine learning-based warning system for mortality risk prediction of patients with COVID-19 was reported, and timely risk stratification using multiple laboratory and clinical factors was improved [32].

Compared with the abovementioned studies, our study has a number of strengths. First, the risk factors for death were analyzed using a nationwide cohort comprising a large number of patients with COVID-19, which resulted in a scoring system that can be widely used for triaging laboratory-confirmed COVID-19 cases. Second, the COPS system was developed using easily accessible information, such as age, underlying disease, dyspnea, mental disturbance, and hemogram parameters. We believe that if a scoring system that uses only simple laboratory tests (eg, hemogram parameters) can be developed and still show good predictability, it will prove to be more cost-effective than systems including other biomarkers such as procalcitonin or cytokine levels. Third, the discriminatory power of our system for predicting death probability was excellent. Finally, this study further analyzed the length of hospital stay according to disease severity, which may assist in preparing medical resources based on patient classification. However, this study is limited by the lack of external verification for our scoring model. Therefore, the current model of overall survival for the diagnosis of COVID-19 would need to be validated in a future cohort.

In conclusion, our study provides a simple scoring system based on information collected at diagnosis for predicting mortality among patients with COVID-19 in a timely manner. Early triaging of patients with COVID-19 using the COPS system can provide new insights for risk-adaptive strategies and optimize the use of medical resources.

\section{Acknowledgments}

We would like to thank Si-Hyun Park and Seunghoon Han for their help with analyzing the data at Korea Disease Control and Prevention Agency. We acknowledge all the health care workers involved in the diagnosis and treatment of patients with COVID-19 in Korea. We thank Korea Disease Control \& Prevention Agency, National Medical Center, and the Health Information Manager in hospitals for their effort in collecting the medical records. This work is funded by the Life Respecting Fund of Seoul St. Mary's hospital and Research Fund of Seoul St. Mary's Hospital, The Catholic University of Korea.

\section{Authors' Contributions}

SYC and SSP wrote the manuscript. SSP and MKS collected and analyzed the data and analyzed it. Drafted manuscript was reviewed by YYB, DGL and DWK. DGL and DWK designed the study, conceived the idea, and planned the project. SYC and SSP contributed equally to this work as first authors. DGL and DWK contributed equally to this work as corresponding authors. All authors approved the final version.

\section{Conflicts of Interest}

None declared.

\section{References}

1. Zhou P, Yang X, Wang X, Hu B, Zhang L, Zhang W, et al. A pneumonia outbreak associated with a new coronavirus of probable bat origin. Nature 2020 Mar;579(7798):270-273 [FREE Full text] [doi: 10.1038/s41586-020-2012-7] [Medline: 32015507]

2. Wang C, Horby PW, Hayden FG, Gao GF. A novel coronavirus outbreak of global health concern. Lancet 2020 Feb 15;395(10223):470-473 [FREE Full text] [doi: 10.1016/S0140-6736(20)30185-9] [Medline: 31986257$]$

3. WHO Coronavirus Disease (COVID-19) Dashboard. World Health Organization. 2020. URL: https://covid19.who.int/ [accessed 2020-11-10]

4. COVID-19 Dashboard by the Center for Systems Science and Engineering (CSSE) at Johns Hopkins University (JHU). Johns Hopkins University \& Medicine - Coronavirus Resource Center. 2020. URL: https://coronavirus.jhu.edu/map.html [accessed 2020-11-10]

5. Guan W, Ni Z, Hu Y, Liang W, Ou C, He J, China Medical Treatment Expert Group for Covid-19. Clinical characteristics of coronavirus disease 2019 in China. N Engl J Med 2020 Apr 30;382(18):1708-1720 [FREE Full text] [doi: 10.1056/NEJMoa2002032] [Medline: 32109013]

6. Gandhi M, Yokoe DS, Havlir DV. Asymptomatic transmission, the Achilles' heel of current strategies to control Covid-19. N Engl J Med 2020 May 28;382(22):2158-2160 [RRE Full text] [doi: 10.1056/NEJMe2009758] [Medline: 32329972] 
7. Long Q, Tang X, Shi Q, Li Q, Deng H, Yuan J, et al. Clinical and immunological assessment of asymptomatic SARS-CoV-2 infections. Nat Med 2020 Aug;26(8):1200-1204. [doi: 10.1038/s41591-020-0965-6] [Medline: 32555424]

8. Cummings MJ, Baldwin MR, Abrams D, Jacobson SD, Meyer BJ, Balough EM, et al. Epidemiology, clinical course, and outcomes of critically ill adults with COVID-19 in New York City: a prospective cohort study. The Lancet 2020 Jun;395(10239):1763-1770. [doi: 10.1016/s0140-6736(20)31189-2]

9. CDC COVID-19 Response Team. Severe Outcomes Among Patients with Coronavirus Disease 2019 (COVID-19) - United States, February 12-March 16, 2020. MMWR Morb Mortal Wkly Rep 2020 Mar 27;69(12):343-346 [FREE Full text] [doi: 10.15585/mmwr.mm6912e2] [Medline: 32214079]

10. Liu D, Cui P, Zeng S, Wang S, Feng X, Xu S, et al. Risk factors for developing into critical COVID-19 patients in Wuhan, China: A multicenter, retrospective, cohort study. EClinicalMedicine 2020 Aug;25:100471 [FREE Full text] [doi: 10.1016/j.eclinm.2020.100471] [Medline: $\underline{\text { 32840491] }}$

11. People with Certain Medical Conditions. Centers for Disease Control and Prevention - COVID-19. URL: https://www. cdc.gov/coronavirus/2019-ncov/need-extra-precautions/people-with-medical-conditions.html [accessed 2021-02-08]

12. Zheng Z, Peng F, Xu B, Zhao J, Liu H, Peng J, et al. J Infect 2020 Aug;81(2):e16-e25 [FREE Full text] [doi: 10.1016/j.jinf.2020.04.021] [Medline: 32335169]

13. Chen R, Liang W, Jiang M, Guan W, Zhan C, Wang T, Medical Treatment Expert Group for COVID-19. Risk factors of fatal outcome in hospitalized subjects with coronavirus disease 2019 from a nationwide analysis in China. Chest 2020 Jul;158(1):97-105 [FREE Full text] [doi: 10.1016/j.chest.2020.04.010] [Medline: 32304772]

14. WHO COVID-19 Case definition. World Health Organization. URL: https://www.who.int/publications/i/item/ WHO-2019-nCoV-Surveillance Case Definition-2020.1 [accessed 2020-11-10]

15. Case definition and people subject to testing (As of June 25, 2020). Coronavirus Disease-19, Republic of Korea. URL: http://ncov.mohw.go.kr/en/baroView.

do?brdId=11\&brdGubun=112\&dataGubun=\&ncvContSeq=\&contSeq=\&board id=\&gubunn [accessed 2020-11-10]

16. Kim ES, Chin BS, Kang CK, Kim NJ, Kang YM, Choi J, Korea National Committee for Clinical Management of COVID-19. Clinical course and outcomes of patients with severe acute respiratory syndrome coronavirus 2 infection: a preliminary report of the first 28 patients from the Korean cohort study on COVID-19. J Korean Med Sci 2020 Apr 06;35(13):e142 [FREE Full text] [doi: 10.3346/jkms.2020.35.e142] [Medline: $\underline{32242348]}$

17. Prognosis score system to predict COVID-19 survival. Young Medical Research - COVID-19 Prognosis Scoring system (COPS). URL: https://ymdtech.kr/covid19-os [accessed 2021-02-08]

18. Nursing Homes and Long-Term Care Facilities. Centers for Disease Control and Prevention - COVID-19. URL: https:/ /www.cdc.gov/coronavirus/2019-ncov/hcp/nursing-home-long-term-care.html [accessed 2020-11-15]

19. Surveillance of COVID-19 at long-term care facilities in the EU/EEA. European Center for Disease Prevention and Control. 2020 May 19. URL: https://www.ecdc.europa.eu/en/publications-data/ surveillance-COVID-19-long-term-care-facilities-EU-EEA [accessed 2021-02-08]

20. McMichael TM, Currie DW, Clark S, Pogosjans S, Kay M, Schwartz NG, Public Health-Seattle and King County, EvergreenHealth, and CDC COVID-19 Investigation Team. Epidemiology of Covid-19 in a long-term care facility in King County, Washington. N Engl J Med 2020 May 21;382(21):2005-2011 [FREE Full text] [doi: 10.1056/NEJMoa2005412] [Medline: $\underline{32220208]}$

21. Kim T, Choi MJ, Kim SB, Kim JY, Lee J, Oh HS, et al. Strategical preparedness and response actions in the healthcare system against coronavirus disease 2019 according to transmission scenario in Korea. Infect Chemother 2020 Sep;52(3):389-395 [FREE Full text] [doi: 10.3947/ic.2020.52.3.389] [Medline: 32757499]

22. Pochon C, Voigt S. Respiratory virus infections in hematopoietic cell transplant recipients. Front Microbiol 2018;9:3294 [FREE Full text] [doi: 10.3389/fmicb.2018.03294] [Medline: $\underline{30687278]}$

23. Huang I, Pranata R. Lymphopenia in severe coronavirus disease-2019 (COVID-19): systematic review and meta-analysis. J Intensive Care 2020;8:36 [FREE Full text] [doi: 10.1186/s40560-020-00453-4] [Medline: 32483488]

24. Chen Z, John Wherry E. T cell responses in patients with COVID-19. Nat Rev Immunol 2020 Sep;20(9):529-536 [FREE Full text] [doi: 10.1038/s41577-020-0402-6] [Medline: 32728222]

25. Coronavirus Disease-19 cases in Korea. Korea Disease Control and Prevention Agency. URL: http://ncov.mohw.go.kr/en/ [accessed 2020-11-10]

26. Zhao Z, Chen A, Hou W, Graham JM, Li H, Richman PS, et al. Prediction model and risk scores of ICU admission and mortality in COVID-19. PLoS One 2020;15(7):e0236618 [FREE Full text] [doi: 10.1371/journal.pone.0236618] [Medline: 32730358]

27. Williamson EJ, Walker AJ, Bhaskaran K, Bacon S, Bates C, Morton CE, et al. Factors associated with COVID-19-related death using OpenSAFELY. Nature 2020 Aug;584(7821):430-436. [doi: 10.1038/s41586-020-2521-4] [Medline: 32640463]

28. Liang W, Liang H, Ou L, Chen B, Chen A, Li C, China Medical Treatment Expert Group for COVID-19. Development and validation of a clinical risk score to predict the occurrence of critical illness in hospitalized patients with COVID-19. JAMA Intern Med 2020 Aug 01;180(8):1081-1089 [FRE Full text] [doi: 10.1001/jamainternmed.2020.2033] [Medline: 32396163] 
29. Bhargava A, Fukushima EA, Levine M, Zhao W, Tanveer F, Szpunar SM, et al. Predictors for severe COVID-19 infection. Clin Infect Dis 2020 Nov 05;71(8):1962-1968 [FREE Full text] [doi: 10.1093/cid/ciaa674] [Medline: $\underline{32472676}$ ]

30. Haimovich AD, Ravindra NG, Stoytchev S, Young HP, Wilson FP, van Dijk D, et al. Development and validation of the quick COVID-19 severity index: a prognostic tool for early clinical decompensation. Ann Emerg Med 2020 Oct;76(4):442-453 [FREE Full text] [doi: 10.1016/j.annemergmed.2020.07.022] [Medline: 33012378]

31. Altschul DJ, Unda SR, Benton J, de la Garza Ramos R, Cezayirli P, Mehler M, et al. A novel severity score to predict inpatient mortality in COVID-19 patients. Sci Rep 2020 Oct 07;10(1):16726 [FREE Full text] [doi: 10.1038/s41598-020-73962-9] [Medline: 33028914]

32. Gao Y, Cai G, Fang W, Li H, Wang S, Chen L, et al. Machine learning based early warning system enables accurate mortality risk prediction for COVID-19. Nat Commun 2020 Oct 06;11(1):5033 [FREE Full text] [doi: 10.1038/s41467-020-18684-2] [Medline: 33024092]

\author{
Abbreviations \\ ALC: absolute lymphocyte count \\ AUROC: area under the receiver operating characteristic curve \\ COPS: COVID-19 prognosis score \\ ECMO: extracorporeal membrane oxygenation \\ KDCA: Korea Disease Control and Prevention Agency
}

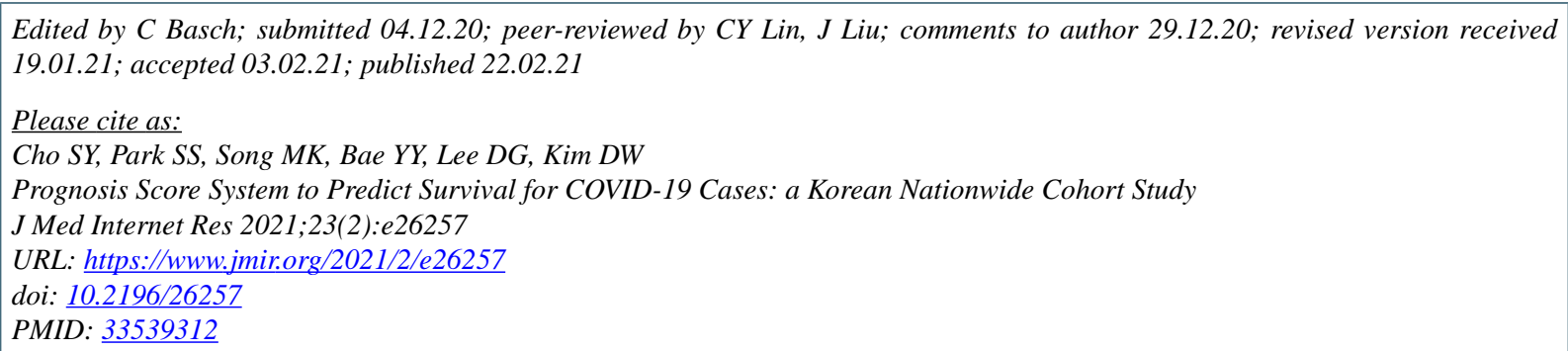

(CSung-Yeon Cho, Sung-Soo Park, Min-Kyu Song, Young Yi Bae, Dong-Gun Lee, Dong-Wook Kim. Originally published in the Journal of Medical Internet Research (http://www.jmir.org), 22.02.2021. This is an open-access article distributed under the terms of the Creative Commons Attribution License (https://creativecommons.org/licenses/by/4.0/), which permits unrestricted use, distribution, and reproduction in any medium, provided the original work, first published in the Journal of Medical Internet Research, is properly cited. The complete bibliographic information, a link to the original publication on http://www.jmir.org/, as well as this copyright and license information must be included. 